\title{
Predictive Value of Neutrophil/Lymphocyte Ratio (NLR) on Cardiovascular Events in Patients with COVID-19
}

\author{
Lili Zhan ${ }^{1, *}$ \\ Yang $\operatorname{Liu}^{2} * *$ \\ Yanxiang Cheng' \\ Weichun Guo ${ }^{2}$ \\ Jing Yang ${ }^{3}$
}

'Department of Gynecology, Renmin Hospital of Wuhan University, Wuhan, 430060, People's Republic of China;

${ }^{2}$ Department of Orthopedics, Renmin Hospital of Wuhan University, Wuhan 430060, People's Republic of China;

${ }^{3}$ Reproductive Medical Center, Renmin Hospital of Wuhan University, Wuhan, 430060, People's Republic of China

*These authors contributed equally to this work
Correspondence: Jing Yang; Weichun Guo No. 99 ZhangZhiDong Street Wuchang District, Wuhan, 430060, Hubei, People's Republic of China

Tel +8627-8804I9II; +8627-8804I9II

Email dryangjing@whu.edu.cn;

guoweichun@aliyun.com
Background: The research on the association between coronavirus disease 2019 (COVID19) and cardiovascular disease (CVD) is still insufficient.

Aim: This study aimed to investigate the association between neutrophil/lymphocyte ratio (NLR) and risk of cardiovascular events in patients with COVID-19.

Methods: Our study included 159 patients with COVID-19 who were measured for NLR value within the first 24 hours of admission. They were followed up for 6 months after discharge and then the relationship between levels of NLR and risk of cardiovascular events was assessed.

Results: In all included patients with COVID-19, NLR values in patients with cardiovascular events [16.28 (4.95-45.18)] were significantly higher than patients without cardiovascular events $[4.75(2.60-7.47)]$. A multivariate logistic regression model revealed that elevated NLR value [increased per SD, 2.41 (1.43-4.29), $P<0.001$; increased 1 of NLR, 2.05 (1.33-4.01), $P=0.010$ ] was significantly and independently associated with increased risk of CVD history on admission after adjustment of related confounding factors. Then, Cox regression analysis revealed that elevated NLR value had a significant association with increased risk of cardiovascular events [increased per SD, 2.36 (1.42-4.36), $P<0.001$; Increased 1 of NLR, 2.00 (1.30-3.97), $P=0.014$ ] after adjustments of these same confounding factors. Furthermore, the ROC curve suggested that NLR value (AUC $=0.803,95 \%$ $\mathrm{CI}=0.731-0.875, P<0.001$, sensitivity $81.2 \%$, and specificity $82.6 \%$ ) has a good predictive value for cardiovascular events during follow-up.

Conclusion: High NLR value was clinically associated with elevated risk of cardiovascular events in patients with COVID-19, which might be a potential biomarker for predicting cardiovascular events in the current COVID-19 pandemic.

Keywords: cardiovascular events, COVID-19, neutrophil/lymphocyte ratio, predictive value, biomarker

\section{Introduction}

Coronavirus disease 2019 (COVID-19) was first reported in Wuhan from China and has rapidly become a global pandemic, causing an unexpected effect on public health issues. ${ }^{1-3}$ The disease is mainly described as respiratory syndromes caused by coronavirus 2 , which is a member of the genus Betacoronavirus. ${ }^{1-4}$ Infection of COVID-19 can trigger and lead to overproduction of pro-inflammatory cytokines and chemokines including interleukin-1 $\beta$ (IL-1 $\beta$ ), interleukin-6 (IL-6), and tumor necrosis factor-a (TNF-a) by the immune system, resulting in multiple 
organ injury. ${ }^{5}$ Cardiovascular diseases (CVDs) have been found among hospitalized patients with COVID-19, and the coexistence of these diseases contributed to a elevated death risk. ${ }^{6-11}$

Existing studies suggested that COVID-19 may be involved in the progress of CVDs, including acute coronary syndrome, myocardial injury, venous thromboembolism, and arrhythmias. ${ }^{12-14}$ It has been also reported that developing hyperinflammatory shock was associated with coronary ischemia and cardiac insufficiency in child patients with COVID-19. ${ }^{15}$ Together, although the exact mechanism has not been fully established, these previous studies demonstrated that an independent association between CVDs and COVID-19 did exist. One thing is clear - the high level of systemic inflammation caused by COVID-19 can deteriorate the progress of cardiovascular damage. ${ }^{5,16-18}$ The inflammatory reaction is an important mechanism involved in the pathogenesis of atherosclerosis and its progression. ${ }^{19}$ Neutrophils, lymphocytes, and related inflammatory mediators can cause vascular damage and play an anti-atherosclerotic role. A large of number studies have suggested that the neutrophil-tolymphocyte ratio (NLR) has been considered as a potential inflammatory biomarker ${ }^{20}$ and even predicts risk of cardiovascular events. Previous studies also suggested that NLR has been associated with thyroiditis, irritable bowel disease, ulcerative colitis, malignancy, and type 2 diabetes mellitus. The association between NLR and CVDs is still not known completely. A previous systematic review also showed that there are prognostic impacts of NLR on predicting cardiovascular events and all-cause mortality among patients with coronary heart disease. ${ }^{21,22}$

However, no recent studies have investigated the association between NLR and cardiovascular events in patients with COVID-19. It is uncertain whether NLR could be a biomarker to predicting cardiovascular events in patients with COVID19. Accordingly, we therefore conducted a longitudinal observation study to explore the association between NLR and risk of cardiovascular events during follow-up of 6 months.

\section{Materials and Methods}

\section{Study Population}

We continuously collected 159 patients with COVID-19 from January 31, 2020 to April 30, 2020 in the Renmin Hospital of Wuhan University. The inclusion criteria were as follows: 1) all patients were diagnosed as COVID-19 according to the diagnostic standard of new coronavirus pneumonia treatment plan (trial version 7$) ;^{23}$ 2) the clinical data of all patients were complete and met the analysis of our study; 3) they all cooperated with the examination and treatment; and 4) they could be followed up after recovery and discharge. According to the severity of COVID-19 on admission, all included patients were divided into mild cases $(\mathrm{N}=143)$ and severe cases $(\mathrm{N}=16)$. Patients with incomplete clinical data $(\mathrm{N}=102)$, in-hospital death $(\mathrm{N}=6)$, history of malignant tumor $(\mathrm{N}=16)$, and other patients $(\mathrm{N}=4)$ who did not meet the inclusion criteria were excluded. For the purpose of this study, after active treatment, all recovered patients $(\mathrm{N}=159)$ were followed up. The Ethics Committee of Renmin Hospital of Wuhan University approved this study and all patients included gave informed consent according to the guidelines of the Declaration of Helsinki.

\section{Follow-Up}

All included patients with COVID-19 were prospectively followed up by telephone two times each month until the occurrence of a composite event (cardiovascular events). The composite events of this study were defined as including stable or unstable angina, myocardial infarction, acute heart failure (HF) or exacerbation of chronic HF, sudden cardiac death, stroke, arrhythmia, and venous thromboembolism. Our reliable and continuous surveillance system can ensure adequate collection of cardiovascular events. For the purpose of this study, as long as one of the composite events occurred, the patient's follow-up was completed. If the current status of these patients was uncertain, referring cardiologists and/or general practitioners were contacted and invited to identify the patient's condition.

\section{Laboratory Measurements}

Venous blood were collected in the first morning after admission. Blood samples were measured for blood routine including red blood cell (RBC), white blood cell (WBC), neutrophil (NEUT), lymphocyte (LY), and others by using an automated blood counter (Sysmex XE5000; Emilio de Azevedo Campos, Porto, Portugal). The alanine transaminase (ALT), aspartate transaminase (AST), low density lipoprotein cholesterol (LDL), and high density lipoprotein cholesterol (HDL) were analyzed by using the Siemens ADVIA 2400 automatic biochemistry analyzer (Siemens AG). The Fasting blood glucose (FBG), albumin (ALB), N-terminal prohormone of B-type natriuretic peptide (NT-proBNP), and hemoglobin $(\mathrm{Hb})$ 
were measured by using a Behring BN ProSpec analyzer (Dade Behring). The CKD Epidemiology Collaboration (CKD-EPI) creatinine was used to calculating the estimated glomerular filtration rate (eGFR). ${ }^{24}$

\section{Statistical Analyses}

The data with normal distributions were expressed as the mean \pm standard deviation (SD). Comparisons between variables with normal distributions were performed by $t$-test The data that were not normally distributed were expressed by median (interquartile range [IQR]) and were analyzed by the Mann-Whitney $U$-test. The distribution of categorical variables was studied using the Chisquare test.

Firstly, to test if NLR value might be associated with CVD history, a corrected logical regression model was performed with NLR value as the independent variable and CVD history as the dependent variable. Secondly, free rate of cardiovascular events was constructed by the Kaplan-Meier method and Log rank test was performed. We will further analyze the association between NLR value and risk of a composite event (cardiovascular events) including stable or unstable angina, myocardial infarction, acute HF or exacerbation of chronic HF, sudden cardiac death, stroke, arrhythmia, and venous thromboembolism during the follow-up of 6 months by using a corrected Cox regression model. Additionally, the predictive value of NLR for the cardiovascular events was determined using receiver operating characteristic (ROC) curves, and area under the curve (AUC), and the 95\% confidence intervals, sensitivity, and specificity were calculated. SPSS 25.0 was used for the statistical analyses. $P \leq 0.05$ was considered as statistically significant.

\section{Results \\ Clinical Characteristics of Patients with COVID-19 After Admission}

The clinical characteristics of all included patients with COVID-19 are presented in Table 1. NLR value in patients with cardiovascular events [16.28 (4.95-45.18)] was significantly higher than patients without cardiovascular events [4.75 (2.60-7.47)]. The patients with cardiovascular events were more likely to be older, current smokers, current drinkers, and had a higher prevalence of hypertension, diabetes, and coronary heart disease. The patients with cardiovascular events also tended to have a higher body mass index (BMI), systolic and diastolic blood pressure. For serological indicators, there was no significant difference between the two groups in liver function (ALT and AST) and renal function ( $\mathrm{SCr}$ and eGFR), as well as in RBC (all $P>0.05$ ). However, significant differences between the two groups existed in $\mathrm{Hb}, \mathrm{ALB}$, HDL, LDL, FBG, NT-proBNP, WBC, NEUT, LY, and NLR, respectively (all $P<0.05$ ).

\section{Elevated NLR Value Had a Significant Association with Increased Risk of CVD History on Admission in Patients with COVID-19}

To confirm the association of NLR value with CVD history on admission in patients with COVID-19, a corrected logistic regression model was performed (Table 2). The multivariate logistic regression model revealed that NLR value [Increased per SD, 2.57 (1.56-4.78), $P<0.001$; Increased 1 of NLR, 2.24 (1.49-4.47), $P<0.001]$ was significantly and independently associated with after adjustment of age and gender was made in Model 1. When BMI was continued to be added into Model 1, our results showed no significant change for the independent association in Model 2 [Increased per SD, 2.51 (1.52-4.53), $P<0.001$; Increased 1 of NLR, 2.20 (1.43-4.31), $P=0.002$ ]. Model 3 [Increased per SD, 2.48 (1.50-4.44), $P<0.001$; Increased 1 of NLR, 2.13 (1.39-4.12), $P=0.006$ ] and Model 4 [Increased per SD, 2.41 (1.43-4.29), $P<0.001$; Increased 1 of NLR, $2.05 \quad(1.33-4.01)$, $P=0.010]$ remained significantly and strongly associated between NLR and CVD history on admission after other variables are corrected.

\section{Elevated NLR Value is Associated with Higher Risk of Cardiovascular Events During Follow-Up in Patients with COVID-19}

Cardiovascular events occurred in 25 patients with COVID-19, including stable or unstable angina $(\mathrm{N}=6)$, myocardial infarction $(\mathrm{N}=7)$, acute $\mathrm{HF}$ or exacerbation of chronic HF $(\mathrm{N}=4)$, sudden cardiac death $(\mathrm{N}=3)$, stroke $(\mathrm{N}=3)$, arrhythmia $(\mathrm{N}=1)$, and venous thromboembolism $(\mathrm{N}=1)$ in patients with COVID-19. Kaplan-Meier analysis in our study demonstrated that patients with a higher NLR value above median (10.14) had a higher rate of cardiovascular events than those with a NLR value below the median value $(P<0.001$, Figure 1$)$. To investigate whether 
Table I Clinical Characteristics of Patients with COVID-19 After Admission

\begin{tabular}{|c|c|c|c|}
\hline Variables & $\begin{array}{l}\text { With Cardiovascular Events } \\
\qquad(\mathrm{N}=\mathbf{2 5})\end{array}$ & $\begin{array}{l}\text { Without Cardiovascular Events } \\
\qquad(\mathrm{N}=134)\end{array}$ & $P$-value \\
\hline Age (years) & $72 \pm 10.3$ & $63.6 \pm 9.3$ & $<0.001$ \\
\hline Gender (male), n (\%) & $12(48.0)$ & $61(45.5)$ & 0.193 \\
\hline BMI $\left(\mathrm{kg} / \mathrm{m}^{2}\right)$ & $26.4 \pm 8.1$ & $23.8 \pm 5.6$ & 0.011 \\
\hline Systolic blood pressure $(\mathrm{mmHg})$ & $148(|39-| 6 \mid)$ & $145(136-158)$ & $<0.22$ \\
\hline Diastolic blood pressure $(\mathrm{mmHg})$ & $86(70-93)$ & $85(7 I-92)$ & 0.306 \\
\hline Current smoker, n (\%) & $16(64.0)$ & $37(27.6)$ & $<0.001$ \\
\hline Current drinker, n (\%) & $5(20.0)$ & $20(14.9)$ & 0.009 \\
\hline $\begin{array}{l}\text { Severity of COVID-I9 } \\
\text { Mild, n (\%) } \\
\text { Severe, n (\%) }\end{array}$ & $\begin{array}{l}15(60.0) \\
10(40.0)\end{array}$ & $\begin{array}{c}128(95.5) \\
6(4.5)\end{array}$ & $<0.001$ \\
\hline $\begin{array}{l}\text { CVD history on admission } \\
\text { Hypertension, n (\%) } \\
\text { Diabetes, n (\%) } \\
\text { Coronary heart disease, n (\%) } \\
\text { Other or unknown, n (\%) }\end{array}$ & $\begin{array}{l}18(72.0) \\
9(36.0) \\
4(16.0) \\
3(12.0)\end{array}$ & $\begin{array}{c}54(40.3) \\
24(17.9) \\
11(8.2) \\
13(9.7)\end{array}$ & $\begin{array}{l}<0.001 \\
<0.001 \\
<0.001 \\
0.084\end{array}$ \\
\hline $\begin{array}{l}\text { Laboratory measurements } \\
\mathrm{SCr}(\mu \mathrm{mol} / \mathrm{L}) \\
\text { eGFR }\left(\mathrm{mL} / \mathrm{min} / 1.73 \mathrm{~m}^{2}\right) \\
\mathrm{Hb}(\mathrm{g} / \mathrm{L}) \\
\mathrm{RBC}\left(10^{12} / \mathrm{L}\right) \\
\mathrm{HDL}(\mathrm{mmol} / \mathrm{L}) \\
\text { LDL }(\mathrm{mmol} / \mathrm{L}) \\
\text { NT-proBNP }(\mathrm{pg} / \mathrm{mL}) \\
\text { FBG }(\mathrm{mg} / \mathrm{dL}) \\
\text { ALB }(\mathrm{g} / \mathrm{L}) \\
\text { ALT }(\mathrm{U} / \mathrm{L}) \\
\text { AST }(0 \mathrm{U} / \mathrm{L}) \\
\text { WBC }\left(10^{9} / \mathrm{L}\right) \\
\text { NEUT }\left(10^{9} / \mathrm{L}\right) \\
\text { LY }\left(10^{9} / \mathrm{L}\right) \\
\text { NLR }\end{array}$ & $\begin{array}{c}85.5(64.9-89.8) \\
86.4(78.7-93.6) \\
106.6 \pm 6.9 \\
3.54 \pm 0.58 \\
1.06 \pm 0.52 \\
2.73 \pm .91 \\
456.3(145.8-684.8) \\
92.7 \pm \mid 4.5 \\
39.6(37.5-42.3) \\
32.7(21.7-41.0) \\
22.0(17.0-41.0) \\
6.8(4.0-10.1) \\
5.4(3.7-9.4) \\
0.4(0.2-1.2) \\
16.28(4.95-45.18)\end{array}$ & $\begin{array}{c}84.9(60.6-79.5) \\
87.6(72.5-90.1) \\
113.9 \pm 5.6 \\
3.29 \pm 0.47 \\
1.55 \pm 0.71 \\
2.21 \pm .64 \\
335.2(102.4-457.4) \\
85.3 \pm 12.4 \\
44.1(40.2-47.2) \\
33.0(15.5-38.0) \\
23.5(40.7-37.2) \\
5.8(4.5-7.3) \\
4.2(2.9-5.8) \\
0.9(0.7-1.1) \\
4.75(2.60-7.47)\end{array}$ & $\begin{array}{l}0.358 \\
0.216 \\
<0.001 \\
0.396 \\
<0.001 \\
<0.001 \\
<0.001 \\
<0.001 \\
<0.001 \\
0.253 \\
0.272 \\
0.021 \\
0.014 \\
0.002 \\
<0.001\end{array}$ \\
\hline
\end{tabular}

Notes: Normally distributed data are presented as the mean \pm SD, non-normally distributed data are presented as the median (interquartile range), and categorical variables are presented as the $n(\%)$.

Abbreviations: COVID-19, coronavirus disease 2019; BMI, body mass index; CVD, cardiovascular disease; SCr, serum creatinine; eGFR, estimated glomerular filtration rate; Hb, hemoglobin; RBC, red blood cell; UA, uric acid; ALB, albumin; ALT alanine transaminase; AST, aspartate transaminase; WBC, white blood cell; HDL, high density lipoprotein; LDL, low density lipoprotein; NT-proBNP, N-terminal prohormone of B-type natriuretic peptide; FBG, fasting blood glucose; NEUT, neutrophil; LY, lymphocyte; NLR, neutrophil-to-lymphocyte ratio.

NLR value was associated with risk of cardiovascular events during follow-up, Cox regression analysis was performed (Table 3). Cox regression analysis revealed that elevated NLR value had a significant association with increased risk of cardiovascular events [Increased per SD, 2.36 (1.42-4.36), $P<0.001$; Increased 1 of NLR, 2.00 (1.30-3.97), $P=0.014]$ after adjustments of age, gender, BMI, current smoker, current drinker, systolic blood pressure, diastolic blood pressure, and laboratory measurements were made in Model 4.

Additionally, a sensitivity analysis was performed by using CVD history as a "covariate" to evaluate the association between NLR and cardiovascular events (Table 4). Importantly, the results still suggested an independent 
Table 2 The Association Between the NLR and Risk of CVD History on Admission in Patients with COVID-19

\begin{tabular}{|l|c|c|c|c|}
\hline Variables & Model I & Model 2 & Model 3 & Model 4 \\
\hline Increased per SD & $2.57(I .56-4.78)$ & $2.5 \mathrm{I}(\mathrm{I} .52-4.53)$ & $2.48(1.50-4.44)$ & $2.4 \mathrm{I}(\mathrm{I} .43-4.29)$ \\
$\boldsymbol{P}$-value & $<0.00 \mathrm{I}$ & $<0.00 \mathrm{I}$ & $<0.00 \mathrm{I}$ & $<0.00 \mathrm{I}$ \\
Increased I of NLR & $2.24(\mathrm{I} .49-4.47)$ & $2.20(\mathrm{I} .43-4.3 \mathrm{I})$ & $2.13(\mathrm{I} .39-4.12)$ & $2.05(\mathrm{I} .33-4.0 \mathrm{I})$ \\
$\boldsymbol{P}$-value & $<0.00 \mathrm{I}$ & 0.002 & 0.006 & 0.010 \\
\hline
\end{tabular}

Notes: Model I: Adjusted for age and gender. Model 2: Adjusted for age, gender, and BMI. Model 3: Adjusted for age, gender, BMI, current smoker, current drinker, and severity of COVID-19. Model 4: Adjusted for age, gender, BMI, current smoker, current drinker, severity of COVID-I9, systolic blood pressure, diastolic blood pressure, and laboratory measurements.

Abbreviations: NLR, neutrophil-to-lymphocyte ratio; COVID-19, coronavirus disease 2019; BMI, body mass index.

association between NLR and cardiovascular events [Increased per SD, $2.21(1.32-5.14), P=0.006$; Increased 1 of NLR, 2.08 (1.28-3.93), $P=0.019]$ in Model 4.

\section{NLR Value Had a Better Prognostic Value for Predicting Cardiovascular Events During Follow-Up in Patients with COVID-19}

To determine the predictive value of NLR for cardiovascular events during follow-up in patients with COVID-19, the area under the receiver operating characteristic curve (ROC-AUC) was performed (Figure 2 and Table 5). The NLR value $(\mathrm{AUC}=0.803,95 \% \mathrm{CI}=0.731-0.875, P<0.001$, sensitivity $81.2 \%$, and specificity $82.6 \%$ ) had a better predictive value than $\mathrm{LY}(\mathrm{AUC}=0.512,95 \% \mathrm{CI}=0.415-0.609$, $P=0.809$, sensitivity $43.6 \%$ and specificity $42.9 \%$ ) and NEUT (AUC $=0.619,95 \%$ CI $=0.525-0.712, P=0.016$, sensitivity $64.4 \%$, and specificity $61.9 \%$ ) for patients with cardiovascular events. These results suggested that NLR value has a good predictive value for risk of cardiovascular events.

\section{Discussion}

We provided the first evidence that increased NLR is associated with higher risk of a composite event (cardiovascular events) including stable or unstable angina, myocardial infarction, acute HF or exacerbation of chronic HF,

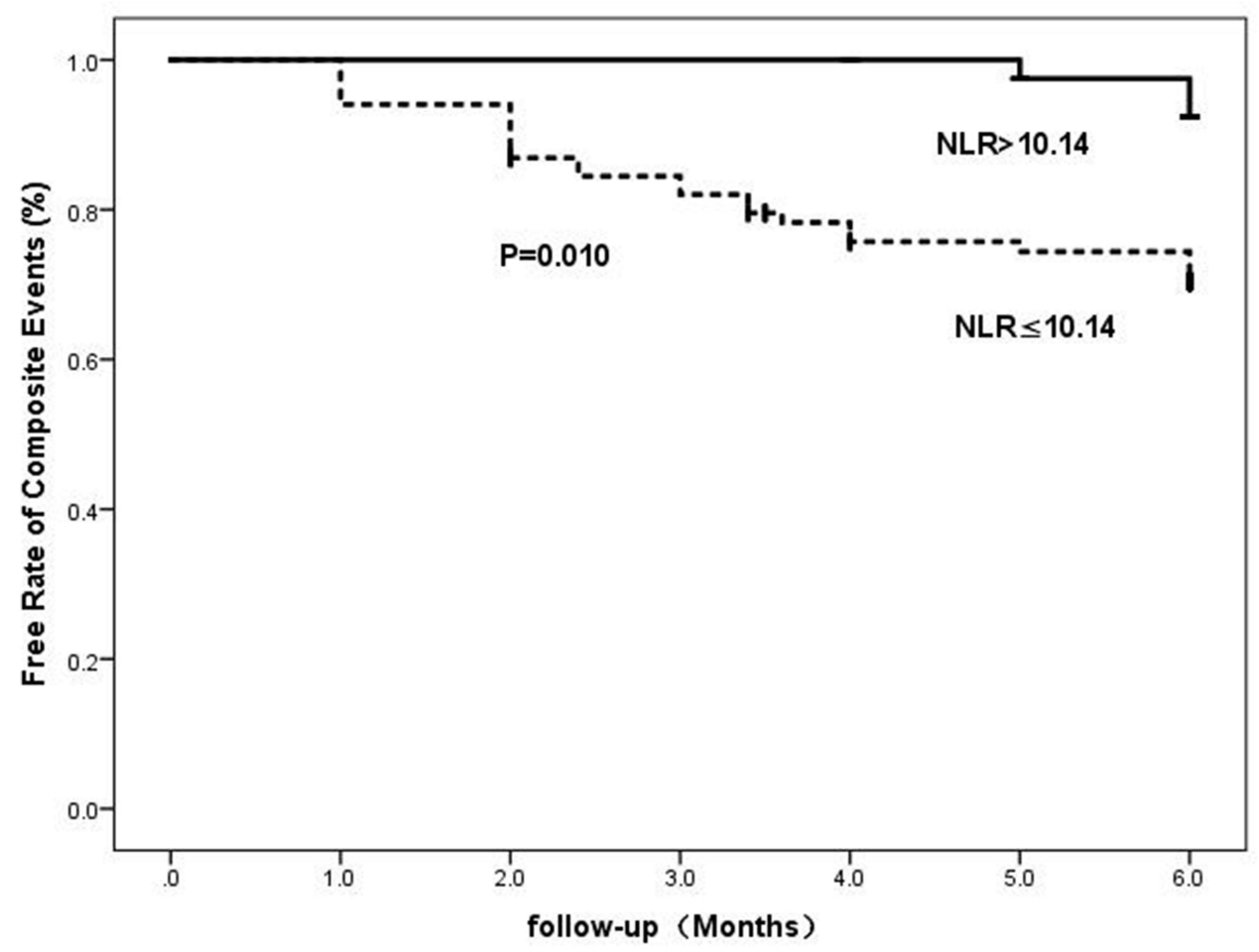

Figure I Kaplan-Meier method and Log rank test for the association between NLR and cardiovascular events during follow-up in patients with COVID-I9. 
Table 3 The Association Between the NLR and Risk of Cardiovascular Events in Patients with COVID-I9 During Follow-UP

\begin{tabular}{|l|c|c|c|c|}
\hline Variables & Model I & Model 2 & Model 3 & Model 4 \\
\hline Per increased SD & $2.43(1.48-5.49)$ & $2.40(1.45-5.42)$ & $2.37(1.43-5.40)$ & $2.36(1.42-4.36)$ \\
P-value & $<0.00 I$ & $<0.001$ & $<0.001$ & $<0.001$ \\
Per increased I of NLR & $2.20(1.43-4.38)$ & $2.16(1.4 I-4.23)$ & $2.04(1.36-4.09)$ & $2.00(1.30-3.97)$ \\
P-value & 0.001 & 0.006 & 0.009 & 0.014 \\
\hline
\end{tabular}

Notes: Model I: Adjusted for age and gender. Model 2: Adjusted for age, gender and BMI. Model 3: Adjusted for age, gender, BMI, current smoker, current drinker and severity of COVID-19. Model 4: Adjusted for age, gender, BMI, current smoker, current drinker, severity of COVID-19, systolic blood pressure, diastolic blood pressure, and laboratory measurements.

Abbreviations: NLR, neutrophil-to-lymphocyte ratio; COVID-19, coronavirus disease 2019; BMI, body mass index.

Table 4 Sensitivity Analysis for the Association Between the NLR and Risk of Cardiovascular Events in Patients with COVID-19

\begin{tabular}{|l|c|c|c|c|}
\hline Variables & Model I & Model 2 & Model 3 & Model 4 \\
\hline Per increased SD & $2.30(1.37-5.37)$ & $2.25(1.34-5.28)$ & $2.23(1.33-5.22)$ & $2.21(1.32-5.14)$ \\
P-value & $<0.001$ & $<0.001$ & 0.002 & 0.006 \\
Per increased I of NLR & $2.14(1.32-4.27)$ & $2.12(1.31-4.19)$ & $2.11(1.29-4.10)$ & $2.08(1.28-3.93)$ \\
P-value & 0.008 & 0.010 & 0.013 & 0.019 \\
\hline
\end{tabular}

Notes: Model I: Adjusted for age and gender. Model 2: Adjusted for age, gender, and BMI. Model 3: Adjusted for age, gender, BMI, current smoker, current drinker, and severity of COVID-19. Model 4: Adjusted for age, gender, BMI, current smoker, current drinker, severity of COVID-19, systolic blood pressure, diastolic blood pressure, and laboratory measurements.

Abbreviations: NLR, neutrophil-to-lymphocyte ratio; COVID-19, coronavirus disease 2019; BMI, body mass index.

sudden cardiac death, stroke, arrhythmia, and venous thromboembolism in patients with COVID-19. NLR might be a potential biomarker for predicting cardiovascular events in patients with COVID-19.

CVDs are a common comorbidity found in patients with severe acute respiratory syndrome (SARS) or middle east respiratory syndrome (MERS), ${ }^{16-18,25,26}$ and similar findings were also observed with COVID-19. ${ }^{16-18,27}$ Early evidence showed that CVDs and CVDs-related risk factors including diabetes mellitus and hypertension were common pre-existing conditions in patients with COVID-19 from China. A early study, including 41 patients from Wuhan who were hospitalized due to COVID-19, showed that diabetes (20\%), hypertension (15\%), and other CVDs $(15 \%)$ were the most common diseases. ${ }^{6}$ The high rate of these comorbidities was also confirmed in subsequent studies. ${ }^{7-11,28-30}$ Importantly, these pre-existing conditions were more likely to exist in critical patients from the intensive care unit (ICU). A study has demonstrated that $31 \%$ of patients had hypertension, $10 \%$ of patients had diabetes, and $15 \%$ of patients had other CVDs in 138 patients infected with COVID-19 hospitalized in the ICU ward. $^{7}$ In the context of these previous studies, finding a modifiable risk factor or predictor that can predict cardiovascular events in patients with COVID-19, so as to detect cardiovascular events early and to further improve the prognosis of patients.
Inflammatory mechanisms may be the best reason for interpretation for the relationship between NLR and high risk of cardiovascular events in patients with COVID-19. Inflammatory cells, such as neutrophils and lymphocytes and their secreted inflammatory mediators are involved in various acute and chronic inflammatory reactions and immune regulation in vivo. ${ }^{31}$ Activated neutrophils can secrete various proteolytic enzymes $^{32}$ and further cause degradation of endothelial damage and basement membrane. ${ }^{33}$ It can infiltrate into atherosclerotic plaques and promote instability of the plaque. ${ }^{34}$ NLR, served as an early inflammatory marker, was associated with progression of atherosclerosis and and its related diseases in various populations. ${ }^{35,36}$ For example, previous studies demonstrated that NLR and C-reactive protein (CRP), serving as the inflammatory biomarkers, have been found to be strongly associated with the risk of CVDs. ${ }^{37-40}$ Previous evidence also suggested that for the ability to predict CVD risk, NLR may be a potential surrogate biomarker of systemic inflammation. ${ }^{41}$ Therefore, we investigated whether NLR is associated with increased of cardiovascular events or can be considered as a modifiable risk factor or predictor that can predict cardiovascular events in patients with COVID-19. Consistent with our hypothesis, our study suggested an independent association of NLR with increased risk of cardiovascular events in 


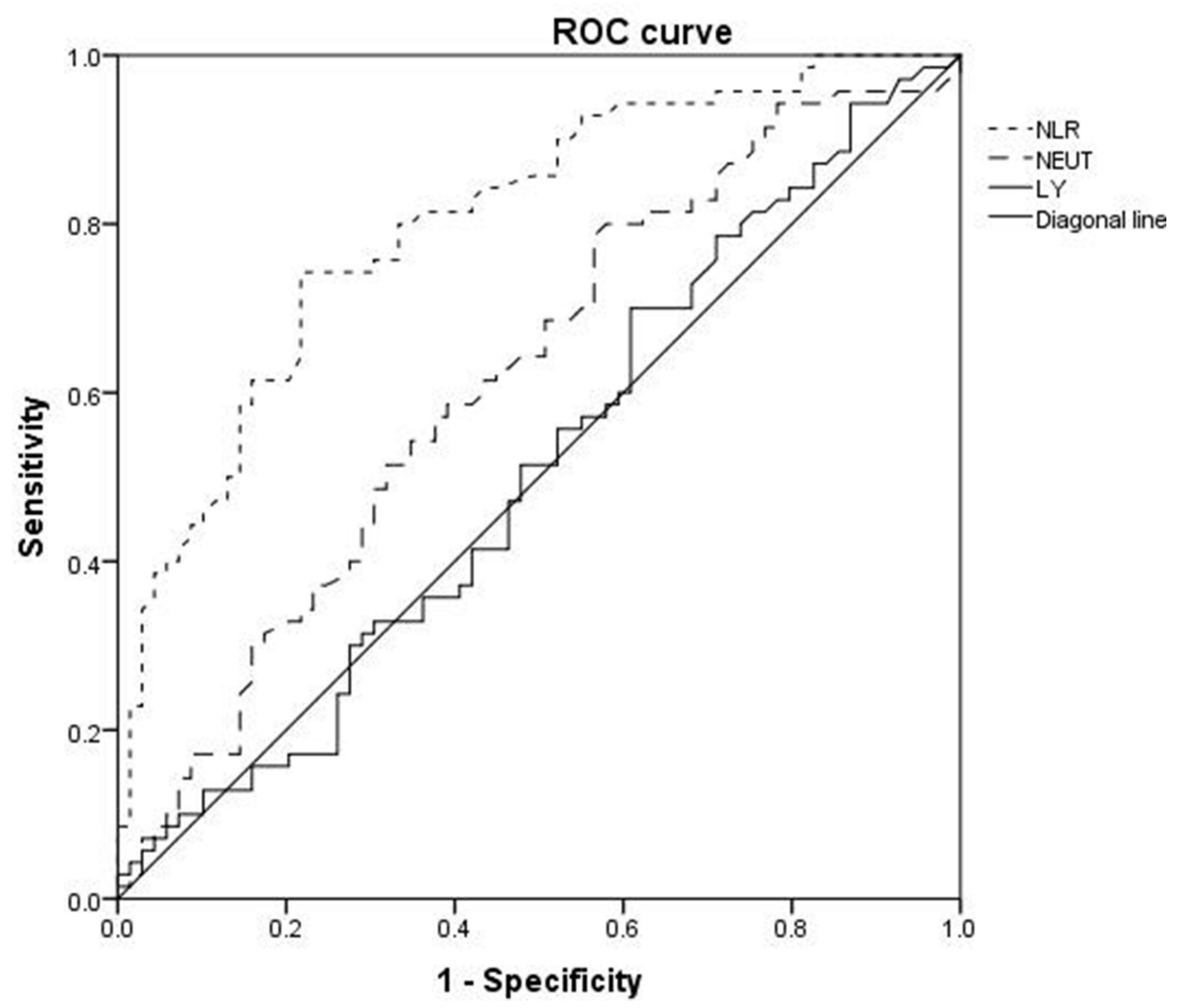

Figure 2 ROC curve for prognostic value of NLR value on predicting cardiovascular events during follow-up in patients with COVID-I9.

patients with COVID-19, which is also supported by previous evidence.

The current study has several strengths. As far as we know, this is the first evidence to investigate the association between NLR and risk of cardiovascular events in patients with COVID-19. Our results suggested an independent predictive value of NLR on detecting cardiovascular events, which can expand previous rare longitudinal study about COVID-19. Furthermore, all components of cardiovascular events consisted of stable or unstable angina, myocardial infarction, acute HF or exacerbation of chronic HF, sudden cardiac death, stroke, arrhythmia, and venous thromboembolism. We comprehensively evaluated the significant relationship between NLR and the risk of cardiovascular events, suggesting that inflammatory mechanisms play an important role in the pathophysiology of CVDs in patients with COVID-19. In addition, sufficient clinical confounding factors were corrected in our analysis, which can ensure that the results were reliable.

Of course, some limitations might be unavoidable. First, although our study is a longitudinal study followed up for about 6 months among patients with COVID-19, the causal link between NLR and risk of cardiovascular events is still unconfirmed because of the short follow-up time and unknown sequence of occurrence time of elevated NLR and cardiovascular events. Future studies need to make clear that NLR increases before cardiovascular events occur in patients with COVID-19. Second, adequate confounding factors correction will lead to over fitting of the model, which may make the correlation between NLR and cardiovascular

Table 5 Diagnostic Ability of NLR Values to Predicting Cardiovascular Events

\begin{tabular}{|l|c|c|c|c|c|}
\hline Variables & AUC & $\mathbf{9 5 \% ~ C l}$ & P-value & Sensitivity (\%) & Specificity (\%) \\
\hline LY $\left(10^{9} / \mathrm{L}\right)$ & 0.512 & $0.415-0.609$ & 0.809 & 43.6 & 42.9 \\
NEUT $\left(10^{9} / \mathrm{L}\right)$ & 0.619 & $0.525-0.712$ & 0.016 & 64.4 & 61.9 \\
NLR & 0.803 & $0.731-0.875$ & $<0.001$ & 81.2 & 82.6 \\
\hline
\end{tabular}

Abbreviations: NEUT, neutrophil; LY, lymphocyte; NLR, neutrophil-to-lymphocyte ratio; AUC, area under the curve. 
events smaller than the actual value. Third, NLR was only detected once within 24 hours of admission and other variables affecting NLR level are complex and unknown. The instability of NLR may cause some bias in our results. Finally, there is also a common limitation that this is a singlecenter study with a small sample number and more studies are needed to identify the value of the NLR on recognizing risk of cardiovascular events in multi-center and largesample studies in the future.

\section{Conclusion}

Our findings provided first evidence that NLR value has a significant association with cardiovascular events in patients with COVID-19. The inflammatory biomarker might be considered a very valuable indicator on predicting cardiovascular events early. Multi-center and largesample studies are necessary to further confirm the reliability of the results in the future.

\section{Acknowledgments}

Lili Zhan and Yang Liu are co-first authors for this study.

\section{Disclosure}

The authors report no conflicts of interest in this work.

\section{References}

1. Roujian L, Xiang Z, Juan L, et al. Genomic characterisation and epidemiology of 2019 novel coronavirus: implications for virus origins and receptor binding. Lancet (London, England). 2020;1022:565-574.

2. Wu F, Zhao S, Yu B, et al. A new coronavirus associated with human respiratory disease in China. Nature. 2020;579(7798):1-8. doi:10.1038/s41586-020-2008-3

3. Zhou P, Yang XL, Wang XG, et al. A pneumonia outbreak associated with a new coronavirus of probable bat origin. Nature. 579:270-273.

4. Hoffmann M, Kleine-Weber H, Schroeder S, et al. SARS-CoV-2 Cell Entry Depends on ACE2 and TMPRSS2 and is blocked by a clinically proven protease inhibitor. Cell. 2020;181(2):271-280. doi:10.1016/j. cell.2020.02.052

5. Tay MZ, Poh CM, Rénia L, et al. The trinity of COVID-19: immunity, inflammation and intervention. Nat Rev Immunol. 2020;20(6):1-12. doi:10.1038/s41577-020-0311-8

6. Ruan Q, Yang K, Wang W, et al. Clinical predictors of mortality due to COVID-19 based on an analysis of data of 150 patients from Wuhan, China. Intensive Care Med. 2020.

7. Huang C, Wang Y, Li X, et al. Clinical features of patients infected with 2019 novel coronavirus in Wuhan, China. Lancet. 2020;395:10223. doi:10.1016/S0140-6736(20)30183-5

8. Zhou F, Yu T, Du R, et al. Clinical course and risk factors for mortality of adult inpatients with COVID-19 in Wuhan, China: a retrospective cohort study. Lancet. 2020;395:10229. doi:10.1016/S0140-6736(20) 30566-3

9. Wang $\mathrm{D}, \mathrm{Hu} \mathrm{B}, \mathrm{Hu} \mathrm{C}$, et al. Clinical Characteristics of 138 Hospitalized Patients With 2019 Novel Coronavirus nfected Pneumonia in Wuhan, China. JAMA. 2020;323:1061. doi:10.1001/ jama.2020.1585
10. Wu Z, Mcgoogan JM. Characteristics of and Important Lessons From the Coronavirus Disease 2019 (COVID-19) Outbreak in China: summary of a Report of 72 Cases From the Chinese Center for Disease Control and Prevention. J Am Med Assoc. 323.

11. Guan WJ, Ni ZY, Hu Y, et al. Clinical characteristics of coronavirus disease 2019 in China. J Integr Med. 2020.

12. Guo T, Fan Y, Chen M, et al. Cardiovascular implications of fatal outcomes of patients with coronavirus disease 2019 (COVID-19). JAMA Cardiol. 2020;5(7):811. doi:10.1001/jamacardio.2020.1017

13. Shi S, Qin M, Shen B, et al. Association of cardiac injury with mortality in hospitalized patients with COVID-19 in Wuhan, China. JAMA Cardiol. 2020;5(7):802. doi:10.1001/jamacardio.2020.0950

14. Shi S, Qin M, Cai Y, et al. Characteristics and clinical significance of myocardial injury in patients with severe coronavirus disease 2019. Eur Heart J. 2020;41(22):2070-2079. doi:10.1093/eurheartj/ehaa408

15. Riphagen S, Gomez X, Martínez CG, et al. Hyperinflammatory shock in children during COVID 19 pandemic. Lancet. 2020;395:1607-1608. doi:10.1016/S0140-6736(20)31094-1

16. Driggin E, Madhavan MV, Bikdeli B, et al. Cardiovascular considerations for patients, health care workers, and health systems during the COVID-19 Pandemic. Elsevier Public Health Emergency Collection. 75(18).

17. Madjid M, Safavi-Naeini P, Solomon SD, et al. Potential effects of coronaviruses on the cardiovascular system: a review. JAMA Cardiol. 2020;5(7). doi:10.1001/jamacardio.2020.1286.

18. Fulchand S. Covid-19 and cardiovascular disease. BMJ (Online). 2020;369:m1997.

19. Wilhelmsen L. Leukocytes and coronary heart disease. Aust N Z J Med. 1980;10(2):135-138. doi:10.1111/j.1445-5994.1980. tb03700.x

20. Venkatraghavan L, Tan TP, Mehta J. et al. Neutrophil Lymphocyte Ratio as a predictor of systemic inflammation - A cross-sectional study in a pre-admission setting. F1000 Res;2015. 4. doi:10.12688/ f1000research.6474.1

21. Wang X, Zhang G, Jiang X, Zhu H, Lu Z, Xu L. Neutrophil to lymphocyte ratio in relation to risk of all-cause mortality and cardiovascular events among patients undergoing angiography or cardiac revascularization: a meta-analysis of observational studies. Atherosclerosis. 2014;234(1):206-213. doi:10.1016/j. atherosclerosis.2014.03.003

22. Giuseppe DL, Monica V. Impact of neutrophils to lymphocytes ratio on major clinical outcomes in patients with acute coronary syndromes: a systematic review and meta-analysis of the literature. Int J Cardiol. 2018;266:31-37. doi:10.1016/j.ijcard.2018.02.116

23. Chen H, Guo J, Wang C, et al. Clinical characteristics and intrauterine vertical transmission potential of COVID-19 infection in nine pregnant women: a retrospective review of medical records. Lancet. 2020;395(10226):809-815. doi:10.1016/S0140-6736(20)30360-3

24. Stevens PE. Evaluation and management of chronic kidney disease: synopsis of the kidney disease: improving global outcomes 2012 clinical practice guideline. Ann Intern Med. 2013;158(11):825. doi:10.7326/0003-4819-158-11-201306040-00007

25. Li SS, Cheng CW, Fu CL, et al. Left ventricular performance in patients with severe acute respiratory syndrome: a 30-day echocardiographic follow-up study. Circulation. 2003;108(15).

26. Peiris J, Chu CM, Cheng V. Clinical progression and viral load in a community outbreak of coronavirus-associated SARS pneumonia: a prospective study. Lancet. 2003;361(9371):1767-1772. doi:10.1016/S0140-6736(03)13412-5

27. Zheng YY, Ma YT, Zhang JY, et al. COVID-19 and the cardiovascular system. Nat Rev Cardiol. 2020;17(5):1-2. doi:10.1038/s41569020-0360-5

28. Grasselli G, Zangrillo A, Zanella A, et al. Baseline Characteristics and Outcomes of 1591 Patients Infected With SARS-CoV-2 Admitted to ICUs of the Lombardy Region, Italy. JAMA. 2020;323(16):1574. doi:10.1001/jama.2020.5394 
29. Goyal P, Choi JJ, Pinheiro LC, et al. Clinical characteristics of COVID-19 in New York City. New Engl J Med. 2020;382 (24):2372-2374. doi:10.1056/NEJMc2010419

30. Jiang F, Deng L, Zhang L, et al. Review of the Clinical Characteristics of Coronavirus Disease 2019 (COVID-19). J Gen Intern Med. 2020;35(5):1545-1549. doi:10.1007/s11606-020-05762W.

31. Onder G, Rezza G, Brusaferro S. Case-Fatality Rate and Characteristics of Patients Dying in Relation to COVID-19 in Italy. JAMA. 2020. doi:10.1001/jama.2020.4683

32. Tavazzi G, Pellegrini C, Maurelli M, et al. Myocardial localization of coronavirus in COVID-19 cardiogenic shock. Eur J Heart Fail. 2020;22(5):911-915. doi:10.1002/ejhf.1828

33. Wichmann D, Sperhake JP, Lütgehetmann M, et al. Autopsy Findings and Venous Thromboembolism in Patients With COVID-19: a Prospective Cohort Study. Ann Intern Med. 2020;173(4).

34. $\mathrm{Xu} \mathrm{Z}$, Shi L, Wang Y, et al. Pathological findings of COVID-19 associated with acute respiratory distress syndrome. Lancet Respir Med. 2020;8(4):420-422. doi:10.1016/S2213-2600(20)30076-X

35. Ahsen A, Ulu MS, Yuksel S, et al. As a new inflammatory marker for familial mediterranean fever: neutrophil-to-lymphocyte ratio. Inflammation. 2013;36(6):1357-1362. doi:10.1007/s10753-0139675-2
36. Günay E, Ulal SS, Akar O, et al. Neutrophil-to-lymphocyte ratio in chronic obstructive pulmonary disease: a retrospective study. Inflammation. 2014;37(2):374-380. doi:10.1007/s10753-013-9749-1

37. Eastin C, Eastin T. Characteristics and Outcomes of 21 Critically Ill Patients with COVID-19 in Washington State. J Emergency Med. 2020;58(4):710.

38. Schaller T, Hirschbühl K, Burkhardt K, et al. Postmortem Examination of Patients with COVID-19. JAMA. 2020;323(24).

39. Jiang F, Yang J, Zhang Y, et al. Angiotensin-converting enzyme 2 and angiotensin 1-7: novel therapeutic targets. Nat Rev Cardiol. 2014;11 (7):413-426. doi:10.1038/nrcardio.2014.59

40. Oudit GY, Kassiri Z, Jiang C, et al. SARS-coronavirus modulation of myocardial ACE2 expression and inflammation in patients with SARS. Eur J Clin Invest. 2010;39(7):618-625. doi:10.1111/j.13652362.2009.02153.x

41. Kwong JC, Schwartz KL, Campitelli MA, et al. Acute myocardial infarction after laboratory-confirmed influenza infection. New Engl J Med. 2018;378(4):345-353. doi:10.1056/NEJMoa1702090
International Journal of General Medicine

\section{Publish your work in this journal}

The International Journal of General Medicine is an international, peer-reviewed open-access journal that focuses on general and internal medicine, pathogenesis, epidemiology, diagnosis, monitoring and treatment protocols. The journal is characterized by the rapid reporting of reviews, original research and clinical studies
Dovepress

across all disease areas. The manuscript management system is completely online and includes a very quick and fair peer-review system, which is all easy to use. Visit http://www.dovepress.com/ testimonials.php to read real quotes from published authors. 